ISSN 1112-9867

\title{
GENERAL DESCRIPTION OF CONFIGURATIONAL COORDINATE MODEL AND KINETIC OF LUMINESCENCE
}

\author{
A. N. Alias, ${ }^{1,}$, Y. Yaacob ${ }^{1}$, Z. M. Zabidi ${ }^{1}$, S. H. N. Alshurdin ${ }^{1}$ and N. A. Aini ${ }^{2}$ \\ ${ }^{1}$ Faculty of Applied Science, UniversitiTeknologi MARA, 35400 Tapah, Perak, Malaysia \\ ${ }^{2}$ Faculty of Applied Science, UniversitiTeknologi MARA, 40450 Shah Alam, Selangor, \\ Malaysia
}

Published online: 17 October 2017

\begin{abstract}
The configuration coordinate model has been used to explain the luminescence behavior of luminescence materials. In order to understand the luminescence process, the configuration coordinate model has explored. We begin by describing some of the early observations and interpretations of optical absorption and of luminescence. The absorption and emission process are involving the optical transition between two harmonic oscillators ground state and excited state. The principal emphasis is on radiative transitions between electronic states, both optical absorption and luminescent emission. Finally, the luminescence-based kinetic determination has examined to study the effect of concentration of excited species to the luminescence process.
\end{abstract}

Keywords: configuration coordinate; luminescence; Franck-Condon.

Author Correspondence, e-mail: ahmadnazib111@perak.uitm.edu.my doi: http://dx.doi.org/10.4314/jfas.v9i5s.40 


\section{INTRODUCTION}

Luminescence is the emission of light by any material after has excited by external energy. The types of luminescence depend upon the source of external energy such as sonoluminescence: produce by excitation of ultrasound wave; photoluminescence: produce by excitation of light; bioluminescence: generation of light from biological activity; cathodoluminescence: produce by excitation of electron; electroluminescence: generation of light from electrical field; chemiluminescence: generation of light from chemical reaction [1]. Typically, the simplest luminescence processes involving the excitation of molecular species from the ground state to the excited state. The emission of light occurs after luminescence species return to the ground state. Luminescence also calls cool body radiation. While, the luminescence material calls phosphor material. Phosphor consists of host crystal and luminescence center or called activator [2]. This activator plays an importance role in the luminescence properties.

The configuration coordinate model has used to explain the luminescence behavior of luminescence materials. Seitz and Mott have introduced this model knew as Mott-Seitz Model [3-4]. In this model, the activators induce the board character of emission and absorption spectra. The emission spectra strongly depend on temperature. While William has explained the luminescence process in solid obeying the Stokes' law that is the absorption energy is much higher compared with the emission energy [5]. In configuration coordinate model, the curves assumed parabolic. This parabolic shape also has been related vibration motion and can be expressed with Hooke's law which can be explaining using harmonic oscillator [6]. The absorption and emission process involves different electronic levels. Franck-Condon principle can be described the transition between two electronic transitions using a simple harmonic oscillator [7]. In this paper, we will explore the harmonic relationship between ground state and excited state of electronic energy levels to describe the transition state. It also allows the prediction of intensity and transition probability parameters such as Einstein coefficient, absorption coefficient oscillator strength and integrated Emission Intensity. 


\section{RESULTS AND DISCUSSION}

\subsection{Overlap Integral}

The harmonic oscillator Schrödinger equation can be solved analytically and the wavefunction is described by

$\psi_{n}(\xi)=\frac{1}{\sqrt{2^{n} n ! \sqrt{\pi}}} H_{n}(\xi) \exp -\left(\xi^{2} / 2\right)$

where $H_{n}(\xi)$ is the Hermite polynomial which given by

$H_{n}(\xi)=(-1)^{n} \exp \xi^{2} \frac{d}{d \xi^{2}} \exp \xi^{2}$

The value of $\mathrm{n}$ in Equation (1) and (2) is the vibration quantum number. $\xi$ is define as the normalized displacement that is $\xi=\gamma \mathrm{r}, \gamma=(\mu \omega / \hbar)^{1 / 2}$ [11]. Fig. 1 shows the harmonic oscillator and wavefunction for the first four state of the harmonic oscillator. The solution for harmonic oscillator shows that the Eigen energy value is positive about the ground state. The vibrational energy separation can write as

$E_{v+1}-E_{v}=\hbar \omega$

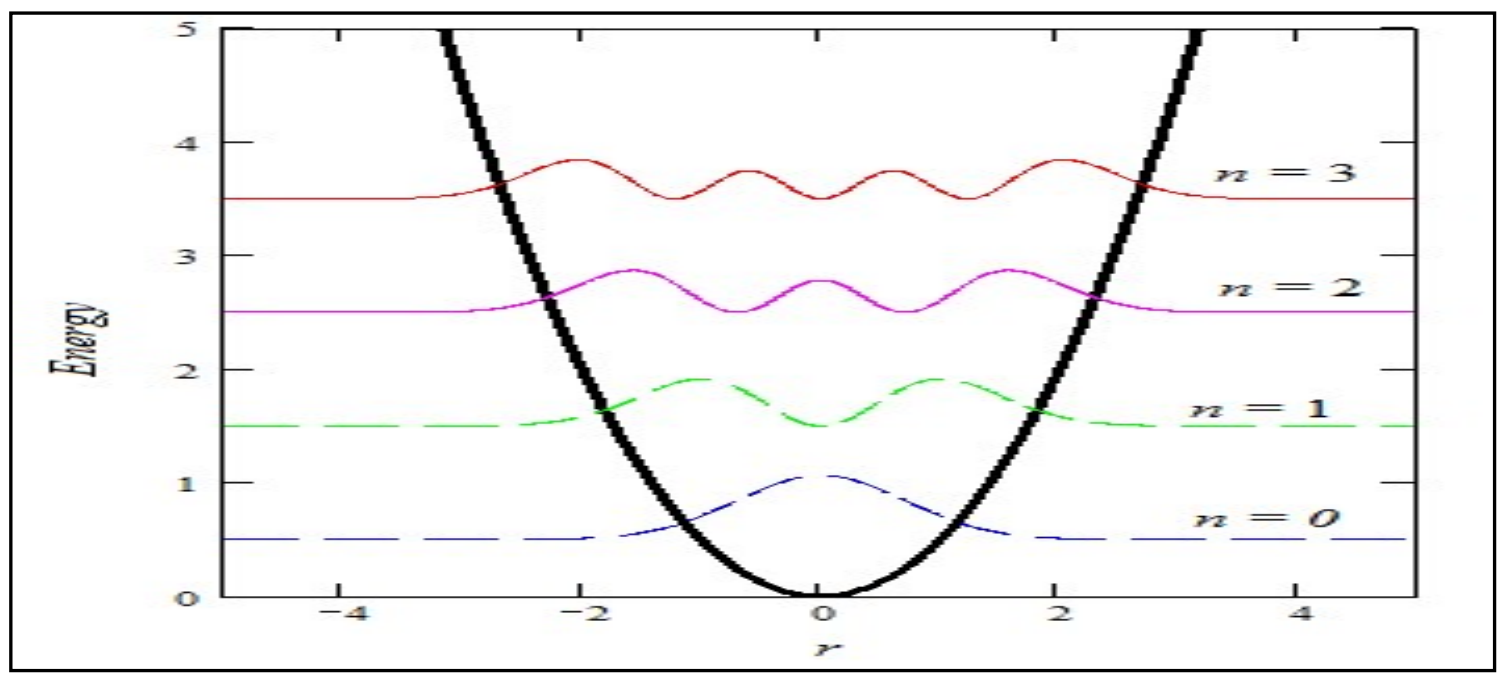

Fig.1. Graphical representation of harmonic oscillator with value of $\mu=1, h=1$ and $k=1$

The absorption spectra are formed from the transition of the ground state to the discrete vibration excited state. From Equation (1), we can write the ground state wavefunction as

$\psi_{g}\left(\xi_{g}\right)=\frac{1}{\sqrt{2^{g} g ! \sqrt{\pi}}} H_{g}\left(\xi_{g}\right) \exp -\left(\xi_{g}^{2} / 2\right)$ 
While, the emission spectra is the transition from the excited state to the ground state. According to the configuration coordinate, the excited state will displace the molecule into small changes of $\Delta r$. Therefore, the normalized displacement become $\xi_{\mathrm{ex}}=\gamma_{\mathrm{ex}}(\mathrm{r}-\Delta \mathrm{r})$. Thus, the excited state wavefunction can write as

$$
\psi_{e x}\left(\xi_{e x}\right)=\frac{1}{\sqrt{2^{e x} g ! \sqrt{\pi}}} H_{e x}\left(\xi_{e x}\right) \exp -\left(\xi_{e x}^{2} / 2\right)
$$

For implementation the transition parameter, $\mathrm{R}_{\mathrm{e}}(\mathrm{r})$ is the electronic transition moment of Equation (6) can be written as

$$
R_{e}(\bar{r})=\int \psi_{g r}^{*}(r) M(r) \psi_{e x}(r) d r
$$

The electronic transition moment is the matrix element of the moment of the electron that is related to the electronic transition integral. The value of $R_{e}(\bar{r})$ depends on the internuclear separation, which can be replaced by min value of electronic transition moment $\bar{r}$ [12]. To fully utilize of Equation (24-28), the Equation (6) can rewrite as [10,12]

$$
S_{g r \rightarrow e x}=R_{e}^{2}(\bar{r}) q_{g r, e x}
$$

with

$$
\bar{r}=\frac{\int \psi_{g r} \psi_{e x} r d r}{\int \psi_{g r} \psi_{e x} d r}
$$

and $q_{g r, e x}$ is the Franck-Condon factor which

$$
q_{g r, e x}=\left|\int \psi_{g r} \psi_{e x} d r\right|^{2}
$$

\subsection{Kinetic of Luminescence}

The emission process is a competitive process based on the formation of the excited state. Jablonski energy level diagram (Fig. 3) is usually used to explain the kinetic of luminescence process. The ground, first and second excited state depicted as $S_{0}, S_{1}$ and $S_{2}$. S denotes as singlet state that the multiplicity of spin quantum number is one. While, $\mathrm{T}_{1}$ is the triplet state which the multiplicity of spin quantum number is equal to three. Within the electronic energy levels, there are discrete vibration energy levels. When the molecule absorbs the energy, the molecule will act to minimize the energy by undergoing vibration relaxation (transition from high vibration energy to the lowest vibration energy) or internal conversion (transition from $\mathrm{S}_{2}$ to $\mathrm{S}_{1}$ ). The emission process can occur either fluorescence or phosphorescence. 
Fluorescence is the radiative transition from $S_{1}$ to $S_{0}$ with a short lifetime. While, phosphorescence is the radiative transition from $T_{1}$ to $S_{0}$ with a longer lifetime.

In most fundamental kinetic of luminescence, the rate of kinetic depends upon the molar absorptivity of the molecule, the intensity of incident light and the concentration of molecular species [14]. The population of molecular species can be describing using Boltzmann distribution which gives by Equation (10)

$$
N_{1}=N_{0} \exp \left(-\frac{\Delta E}{k T}\right)_{(10)}
$$

where $\mathrm{N}$ is the number of molecules in the state 1 and $0, \Delta \mathrm{E}$ is the energy differences between state 1 and $0, \mathrm{k}$ is the Boltzmann constant and $\mathrm{T}$ is the temperature.

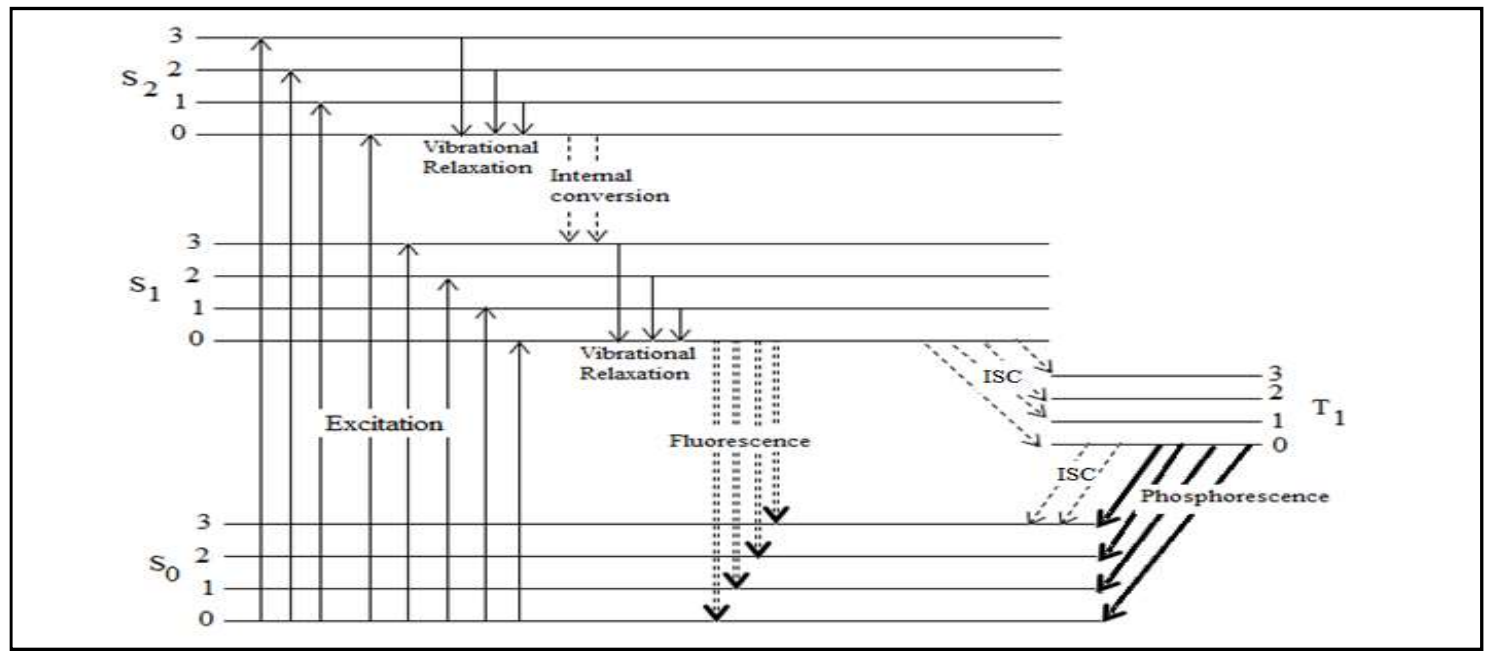

Fig.3.Jablonski diagram for the photophysical processes of fluorescence and phosphorescence.

ISC means intersystem crossing. Redrawn and adapted from [13]

In photochemical reaction, the light will be considered as one of the reactants. Under steady-state irradiation, the kinetic mechanisms involved are given in Table 1. In this kinetic mechanism, we are not considered the triplet-triplet annihilation and bimolecular triplet quenching. The rate of formation of the excited state can be linked using steady or stationary state that is;

$\frac{d\left[S_{1}\right]}{d t}=E_{0}-\left[k_{1}^{*}+k_{1}+k_{2}+k_{Y}\right] S_{1}=0$ 
Table 1. Rate reaction process

\begin{tabular}{|c|c|c|}
\hline Process & Description & Rate \\
\hline$S_{0}+\hbar \omega \stackrel{E_{a b s}}{\rightarrow} S_{1}$ & Absorption process & $\mathrm{E}_{\mathrm{abs}}$ \\
\hline$S_{1} \stackrel{k_{1}^{*}}{\rightarrow} S_{0}+\hbar \omega_{F}$ & Fluorescence & $\mathrm{k}_{1}^{*}$ \\
\hline$S_{1} \stackrel{k_{1}}{\rightarrow} S_{0}$ & Fluorescence quenching & $\mathrm{k}_{1}$ \\
\hline$S_{1} \stackrel{k_{2}}{\rightarrow} T_{1}$ & intersystem crossing & $\mathrm{k}_{2}$ \\
\hline$S_{1} \stackrel{k_{Y}}{\rightarrow}$ product & photochemical reaction, product yield & $\mathrm{k}_{\mathrm{Y}}$ \\
\hline$T_{1} \stackrel{k_{3}}{\rightarrow} S_{0}$ & phosphorescence quenching & $\mathrm{k}_{3}$ \\
\hline$T_{1} \stackrel{k_{3}^{*}}{\rightarrow} S_{0}+\hbar \omega_{P}$ & phosphorescence emission & $\mathrm{k}_{3} *$ \\
\hline$T_{1}+\hbar \omega_{1} \stackrel{g_{1}}{\rightarrow} T_{i}$ & triplet-triplet absorption, $\mathrm{T}_{\mathrm{i}}$ is higher than $\mathrm{T}_{1}$ & $\mathrm{~g}_{1}$ \\
\hline$T_{i} \stackrel{g_{2}}{\rightarrow} T_{1}$ & vibration Relaxation & $\mathrm{g}_{1}$ \\
\hline$T_{1} \stackrel{k_{y}^{\prime}}{\rightarrow}$ product & photochemical reaction, product yield & $\mathrm{k}_{\mathrm{Y}}$ \\
\hline
\end{tabular}

For the fluorescence emission,

$\phi_{F}=\frac{\left[S_{1}\right] k_{1}^{*}}{E_{0}}$

From Equation (11) and (12), the fluorescence quantum yield can be equated as

$\phi_{F}=\frac{k_{1} *}{k_{1} *+k_{1}+k_{2}+k_{Y}}$

Equation (13) shows that the fluorescence quantum yield is merely been the ratio of rate constants. The non-radiative emission and rate of product is a competitor for the fluorescence emission. For investigating the excitation population after the molecule has been excited (the light turn off), the excited state population is given by

$-\frac{d\left[S_{1}\right]}{d t}=\left[k_{1}^{*}+k_{1}+k_{2}+k_{Y}\right] S_{1}$

If we integral Equation (14), the equation become

$\left[S_{1}\right]_{t}=\left[S_{1}\right]_{0} \exp \left(-\frac{t}{\tau_{f}}\right)$ 
Equation (15) shows the excited state decay exponentially with factor ${ }^{\tau_{f}}$. The value of ${ }^{\tau_{f}}$ can be written as

$$
\tau_{f}^{-1}=k_{1}^{*}+k_{1}+k_{2}+k_{Y}
$$

Next, we consider the photophysical process for triplet state. Phosphorescence is the relaxation of the excited state with different spin multicity. The recombination time in the range of microsecond to second. Based on Table 1, the population rate for triplet state $T_{1}$ is

$$
\frac{d\left[T_{1}\right]}{d t}=g_{2}\left[T_{i}\right]-g_{1}\left[T_{1}\right]-\left(k_{3} *+k_{3}+k_{Y}^{\prime}\right)\left[T_{1}\right]+k_{2}\left[S_{1}\right]
$$

While, the high level triplet state $\mathrm{T}_{\mathrm{i}}$.

$$
\frac{d\left[T_{i}\right]}{d t}=g_{i}\left[T_{1}\right]-\left(g_{2}+g_{3}\right)\left[T_{i}\right]
$$

For the steady state condition, the rate of high level triplet state is equal to zero. Therefore, Equation (18) can rearrange to become Equation (19).

$$
\left[T_{i}\right]=\frac{g_{1}\left[T_{1}\right]}{\left(g_{2}+g_{3}\right)}
$$

Substitute Equation (19) into (17) and let $\beta=\mathrm{k}_{2}\left[\mathrm{~S}_{1}\right]$. Then, we integral Equation (19) from $\mathrm{t}^{\prime}=$ 0 until $t^{\prime}=t$. Therefore, the solution of can be written as

$$
\left[T_{1}\right](t)=\left[T_{1}\right]_{0} \exp (-\gamma t)+\frac{\beta}{\gamma}(1-\exp (-\gamma t))
$$

This equation shows that the emission of light is proportional to the number of excited population species. The phosphorescence strongly depends upon the number of population in singlet state which experience for intersystem crossing.

\section{MATHEMATICAL MODELLING FOR THE LUMINESCENCE PROCESS}

To understand the luminescence process, the configuration coordinate model has explored. The absorption and emission processes are involving the optical transition between the harmonic oscillator from the ground state and excited state. Finally, implementation to transition parameter was performing to the adequate explanation of luminescence process. 


\subsection{Theory of Configuration Coordinate}

Configuration coordinate model emphasizes dislocation of ions from the equilibrium. Diagram of energy vs configuration coordinate $r$ (Fig. 2) has been used to describe the transition and rearrangement of ions (luminescence center). The rearrangement of ions during the excitation induces the small changes of $\Delta r$. The value of $\Delta r$ strongly depends upon the size and the charge of the host lattice and activator [8]. According to Frank-Condon principle, in any optical transition, the atomic nuclei remain at rest. Therefore, the luminescence center can absorb the energy equal to $E_{x}$. Next, the relaxation occurs. The energy difference between two energies valus of $r_{o}$ and $\Delta r$ is the energies of offset that is equal to $S_{0} \hbar \omega$.

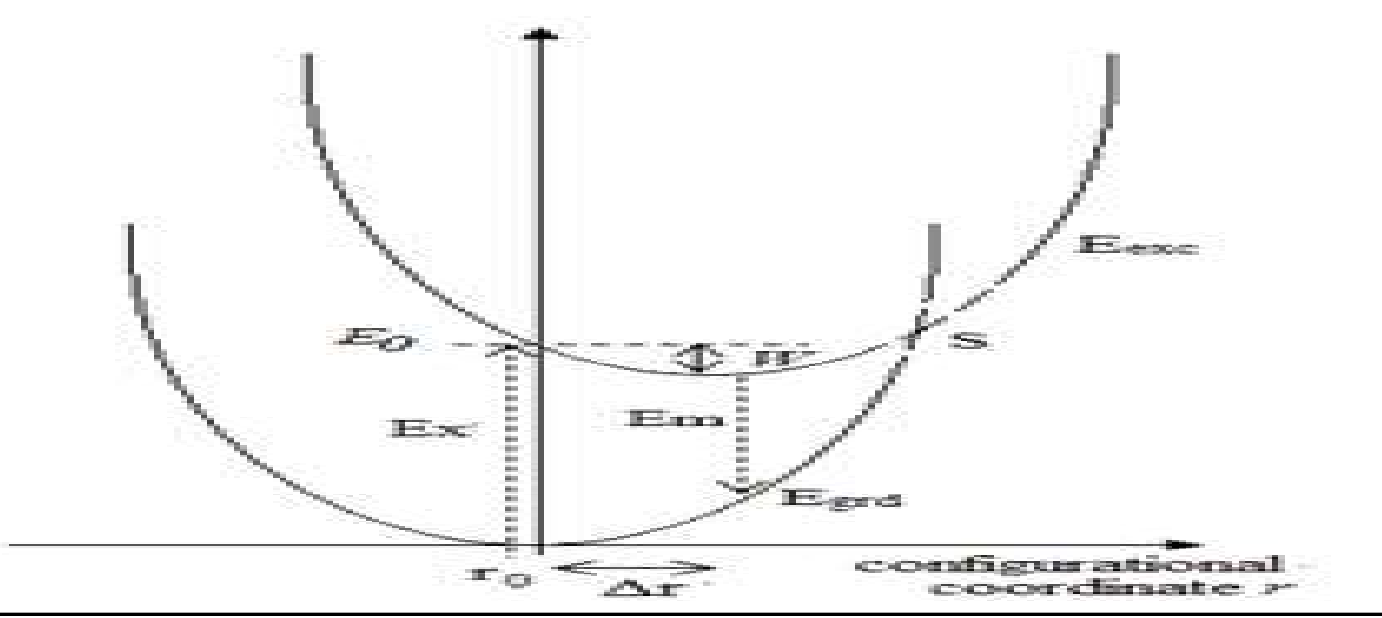

Fig.2. Schematic configuration coordinate diagram for excitation (Ex) and emission (Em) transitions

\subsection{Eigen Values of the Harmonic Oscillator}

The Harmonic oscillator provides the interaction of vibrational of the molecule. When the molecule absorbs the energy, the molecule will excite and displace from the equilibrium $\Delta \mathrm{r}$. The potential energy are related to force by

$$
\frac{d V(r)}{d r}=k r
$$

where $\mathrm{k}$ is the proportional constant that related with bonding force. Therefore,

$$
V(r)=\frac{1}{2} k r^{2}
$$

If we substitute equation (22) into the Schrödinger equation yield 
$-\frac{\hbar^{2}}{2 \mu} \frac{d^{2} \psi(r)}{d r^{2}}+\frac{1}{2} k r^{2} \psi(r)=E \psi(r)$

where ${ }^{\mu}$ is the effective mass and $\hbar$ is the Planck constant. The derivation of Eigen value of Harmonic oscillator can be found in the literature [9].

\subsection{Implementation of Transition Parameters}

This When the electromagnetic radiation interacts with the molecule, the oscillating electric field of radiation may cause the perturbation in the potential energy of the molecule. In non-magnetic medium, the optical transition probability parameters are listed in Equation (24-28)

Einstein B-coefficient, ${ }^{B_{g r \rightarrow e x}} \propto S_{g r \rightarrow e x}$

Absorption coefficient, $k_{g r \rightarrow e x} \propto v_{g r, e x} S_{g r \rightarrow e x}$

Oscillator strength, $f_{g r \rightarrow e x} \propto v_{g r, e x}^{2} S_{g r \rightarrow e x}$

Einstein A- coefficient, $A_{g r \rightarrow e x} \propto v_{g r, e x}^{3} S_{g r \rightarrow e x}(27)$

Integrated Emission Intensity $I_{g r \rightarrow e x} \propto v_{g r, e x}^{4} S_{g r \rightarrow e x}$

The $v_{e, g}$ is the characteristic of band frequency [10]. All the optical parameters are directly proportional to the wavefunction overlap between ground state and excited state. This overlap integral is given by,

$S_{g r \rightarrow e x}=\int \psi_{g r}(r) R_{e}(r) \psi_{e x}(r) d r$

where $\mathrm{R}_{\mathrm{e}}(\mathrm{r})$ is the electronic transition moment.

\section{CONCLUSION}

The one-dimensional configurational coordinate diagram is a useful tool for describing absorption and emission phenomena can merely lead to approximate results. Theoretically, this model is valid if and only if the interaction of the molecule involves, especially in vibrational mode. The effect of the either electron spins multiplicity can lead to the fluorescence or phosphorescence emission which strongly depends on the concentration species. 


\section{ACKNOWLEDGEMENTS}

This work was funded by 600-RMI/RAGS 5/3 (8/2015) iRAGS and 600-IRMI/FRGS 5/3 (88/2016) FRGS. The authors would like to thank Faculty of Applied Sciences, UiTM Perak and Ministry of Higher Education for supporting this project.

\section{REFERENCES}

[1] Khand I U, Lanez T, Pauson P L. Ferrocene derivatives. Part 24. Synthesis of dihydro-2-pyrindines and dihydro-3 H-2-cyclopent [c] azepines by photolysis of their cyclopentadienyliron derivatives. Journal of the Chemical Society, Perkin Transactions 1, 1989(11):2075-2078

[2] VijD. R. Luminescence of solids. Berlin: Springer Science and Business Media, 2012

[3] Zhou S, Yin L. CdS quantum dots sensitized mesoporousBiVO 4 heterostructures for solar cells with enhanced photo-electrical conversion efficiency. Journal of Alloys and Compounds, 2017, 691:1040-1048

[4] Seitz F. Low temperature luminescence of inorganic solids. Transactions of the Faraday Society, 1939, 35:79-87

[5] Mott N. F., Davis E. A. Electronic processes in non-crystalline materials. England: Oxford University Press, 2012

[6] Johnson P D, Williams F E. Simplified configuration coordinate model for KCl: Tl. Physical Review, 1960, 117(4):964-969

[7] Murthy K V, Virk H S. Luminescence phenomena: An introduction. Defect and Diffusion Forum 2014, 347:1-34

[8] Wright J C, Zielinski T J. Franck-Condon factors and their use in undergraduate quantum mechanics. Journal of Chemical Education, 1999, 76(10):1367-1373

[9] Blasse G. Fluorescence of niobium-activated antimonates and an empirical criterion for the occurrence of luminescence. Journal of Chemical Physics, 1968, 48(7):3108-3114 [10] Basdevant J. L. Lectures on quantum mechanics: With problems, exercises and their solutions. Berlin: Springer, 2016

[11] Nicholls RW. The interpolation of Franck-Condon factor arrays for molecular band systems. Journal of Quantitative Spectroscopy andRadiative Transfer. 1965, 5(5):647-667 
[12] Ghatak A. K., Lokanathan S. Quantum mechanics: Theory and applications. Berlin: Springer Verlag, 2004

[13]Fraser P A, Jarmain W R. Vibrational transition probabilities of diatomic molecules: III. Proceedings of the Physical Society. Section A, 1953, 66(12):939-941

[14] Alias A N, Zabidi Z M, Ali A M, Harun M K, Yahya M Z. Optical characterization and properties of polymeric materials for optoelectronic and photonic applications. International Journal of Applied Science and Technology, 2013, 3(5):11-38

\section{How to cite this article:}

Alias A N, Yaacob Y, Zabidi Z M, Alshurdin S H N, Aini N A. General description of configurational coordinate model and kinetic of luminescence. J. Fundam. Appl. Sci., 2017, 9(5S), 568-578. 